PROCEEDINGS OF THE

AMERICAN MATHEMATICAL SOCIETY

Volume 137, Number 4, April 2009, Pages 1381-1388

S 0002-9939(08)09733-5

Article electronically published on October 20, 2008

\title{
LINEAR ISOMETRIES BETWEEN SPACES OF VECTOR-VALUED LIPSCHITZ FUNCTIONS
}

\author{
A. JIMÉNEZ-VARGAS AND MOISÉS VILLEGAS-VALLECILLOS
}

(Communicated by Nigel J. Kalton)

\begin{abstract}
In this paper we state a Lipschitz version of a theorem due to Cambern concerning into linear isometries between spaces of vector-valued continuous functions and deduce a Lipschitz version of a celebrated theorem due to Jerison concerning onto linear isometries between such spaces.
\end{abstract}

\section{INTRODUCTION}

Given a metric space $(X, d)$ and a Banach space $E$, we denote by $\operatorname{Lip}(X, E)$ the Banach space of all bounded Lipschitz functions $f: X \rightarrow E$ with the norm $\|f\|=\max \left\{L(f),\|f\|_{\infty}\right\}$, where

$$
L(f)=\sup \{\|f(x)-f(y)\| / d(x, y): x, y \in X, x \neq y\} .
$$

If $E$ is the field of real or complex numbers, we shall write simply $\operatorname{Lip}(X)$.

The study of surjective linear isometries between spaces $\operatorname{Lip}(X)$ was initiated by Roy 9] and Vasavada [10. In [9, Theorem 1.7], Roy proved that if $(X, d)$ is a compact connected metric space with diameter at most 1 , then a map $T$ is a surjective linear isometry from $\operatorname{Lip}(X)$ onto itself if and only if there exist a surjective isometry $\varphi: X \rightarrow X$ and a scalar $\tau$ of modulus 1 such that

$$
T(f)(y)=\tau f(\varphi(y)), \quad \forall y \in Y, \forall f \in \operatorname{Lip}(X) .
$$

In [8, Theorem 2], Novinger improved slightly Roy's result by considering linear isometries from $\operatorname{Lip}(X)$ onto $\operatorname{Lip}(Y)$. Vasavada [10] proved it for linear isometries from $\operatorname{Lip}(X)$ onto $\operatorname{Lip}(Y)$ when the metric spaces $X, Y$ are compact with diameter at most 2 and $\beta$-connected for some $\beta<1$. Weaver [1] developed a technique to remove the compactness assumption on $X$ and $Y$ and showed that the above-mentioned characterization holds if $X, Y$ are complete and 1-connected with diameter at most 2 [11, Theorem D]. The reduction to metric spaces of diameter at most 2 is not restrictive since if $(X, d)$ is a metric space and $X^{\prime}$ is the set $X$ remetrized with the metric $d^{\prime}(x, y)=\min \{d(x, y), 2\}$, then the diameter of $X^{\prime}$ is at most 2 and $\operatorname{Lip}\left(X^{\prime}\right)$ is isometrically isomorphic to $\operatorname{Lip}(X)$ [12, Proposition 1.7.1]. We must also mention the complete research carried out on surjective linear isometries between spaces of Hölder functions [2, 3, 6, 7]. We refer the reader to Weaver's

Received by the editors April 28, 2008.

2000 Mathematics Subject Classification. Primary 46B04, 46E40; Secondary 46E15.

Key words and phrases. Banach-Stone theorem, linear isometry, Lipschitz function.

The first author was partially supported by Junta de Andalucía grants FQM-1215 and FQM1438, and by MEC grant MTM2006-4837. 
book Lipschitz Algebras 12 for unexplained terminology and more information on the subject. This is essentially the history of the onto scalar-valued case. Recently, into linear isometries (that is, not necessarily surjective) and codimension 1 linear isometries between spaces $\operatorname{Lip}(X)$ have been studied in [5].

In this note we shall go a step further and give a complete description of linear isometries between spaces of vector-valued Lipschitz functions. To our knowledge, little or nothing is known on the matter in the vector-valued case. Our approach to the problem is not based on extreme points as in all aforementioned papers. We have used here a different method which is influenced by that utilized by Cambern 1 to characterize into linear isometries between spaces $C(X, E)$ of continuous functions from a compact Hausdorff space $X$ into a Banach space $E$ with the supremum norm. In [4], Jerison extended to the vector case the classical Banach-Stone theorem about onto linear isometries between spaces $C(X)$, and Jerison's theorem was generalized by Cambern [1] by considering into linear isometries.

The aim of this paper is to show that Cambern's and Jerison's theorems have a natural formulation in the context of Lipschitz functions.

\section{A Lipschitz version of CAMBern's theorem}

We begin by introducing some notation. Given a Banach space $E, S_{E}$ will denote its unit sphere and $B_{E}$ its closed unit ball. Let us recall that a Banach space $E$ is said to be strictly convex if every element of $S_{E}$ is an extreme point of $B_{E}$. For Banach spaces $E$ and $F, L(E, F)$ will stand for the Banach space of all bounded linear operators from $E$ into $F$ with the canonical norm of operators. In the case $E=F$, we shall write $L(E)$ instead of $L(E, F)$. Given a metric space $(X, d)$, we shall denote by $1_{X}$ the function constantly 1 on $X$ and by $\operatorname{diam}(X)$ the diameter of $X$. If $\varphi: X \rightarrow Y$ is a Lipschitz map between metric spaces, $L(\varphi)$ will be its Lipschitz constant.

For any $f \in \operatorname{Lip}(X)$ and $e \in E$, define $f \otimes e: X \rightarrow E$ by $(f \otimes e)(x)=f(x) e$. It is easy to check that $f \otimes e \in \operatorname{Lip}(X, E)$ with $\|f \otimes e\|_{\infty}=\|f\|_{\infty}\|e\|$ and $L(f \otimes e)=$ $L(f)\|e\|$, and thus $\|f \otimes e\|=\|f\|\|e\|$.

Theorem 2.1. Let $X$ and $Y$ be compact metric spaces and let $E$ be a strictly convex Banach space. Let $T$ be a linear isometry from $\operatorname{Lip}(X, E)$ into $\operatorname{Lip}(Y, E)$ such that $T\left(1_{X} \otimes e\right)=1_{Y} \otimes e$ for some $e \in S_{E}$. Then there exists a Lipschitz map $\varphi$ from a closed subset $Y_{0}$ of $Y$ onto $X$ with $L(\varphi) \leq \max \{1, \operatorname{diam}(X) / 2\}$, and a Lipschitz map $y \mapsto T_{y}$ from $Y$ into $L(E)$ with $\left\|T_{y}\right\|=1$ for all $y \in Y$, such that

$$
T(f)(y)=T_{y}(f(\varphi(y))), \quad \forall y \in Y_{0}, \forall f \in \operatorname{Lip}(X, E) .
$$

Proof. For each $x \in X$, define

$$
F(x)=\left\{f \in \operatorname{Lip}(X, E): f(x)=\|f\|_{\infty} e\right\} .
$$

Clearly, $1_{X} \otimes e \in F(x)$. For each $\delta>0$, the map $h_{x, \delta} \otimes e: X \rightarrow E$, defined by

$$
h_{x, \delta}(z)=\max \{0,1-d(z, x) / \delta\} \quad(z \in X),
$$

belongs to $F(x)$. Indeed, an easy verification shows that $h_{x, \delta} \in \operatorname{Lip}(X)$ with $\left\|h_{x, \delta}\right\|_{\infty}=1=h_{x, \delta}(x)$. Hence $h_{x, \delta} \otimes e \in \operatorname{Lip}(X, E)$ with $\left\|h_{x, \delta} \otimes e\right\|_{\infty}=1$ and $\left(h_{x, \delta} \otimes e\right)(x)=e$. Then $\left(h_{x, \delta} \otimes e\right)(x)=\left\|h_{x, \delta} \otimes e\right\|_{\infty} e$ and thus $h_{x, \delta} \otimes e \in F(x)$.

We shall prove the theorem in a series of steps. 
Step 1. Let $x \in X$. For each $f \in F(x)$, the set

$$
P(f)=\{y \in Y: T(f)(y)=f(x)\}
$$

is nonempty and closed.

Let $f \in F(x)$. If $f=0$, then $P(f)=Y$ and there is nothing to prove. Suppose $f \neq 0$ and consider $g=\|f\|_{\infty} f+\|f\|^{2}\left(1_{X} \otimes e\right)$. Clearly, $g \in \operatorname{Lip}(X, E)$ with $L(g)=\|f\|_{\infty} L(f)$ and $g(x)=\left(\|f\|_{\infty}^{2}+\|f\|^{2}\right) e$. The latter equality implies $g \neq 0$. Since

$$
L(g) \leq\|f\|_{\infty}\|f\| \leq\|f\|_{\infty}^{2}+\|f\|^{2}=\|g(x)\| \leq\|g\|_{\infty}
$$

it follows that $\|g\|=\|g\|_{\infty}$. Moreover, $\|g\|_{\infty}=\|g(x)\|=\|f\|_{\infty}^{2}+\|f\|^{2}$ since

$$
\|g\|_{\infty}=\|\| f\left\|_{\infty} f+\right\| f\left\|^{2}\left(1_{X} \otimes e\right)\right\|_{\infty} \leq\|f\|_{\infty}^{2}+\|f\|^{2}=\|g(x)\| .
$$

We now claim that there exists a point $y \in Y$ such that $T(g /\|g\|)(y)=e$. Contrary to our claim, assume $e \neq T(g /\|g\|)(y)$ for all $y \in Y$. Let $\varepsilon>0$ and take $h=$ $g /\|g\|+\varepsilon\left(1_{X} \otimes e\right)$. Clearly, $h \in \operatorname{Lip}(X, E)$ and $T(h)=T(g) /\|g\|+\varepsilon\left(1_{Y} \otimes e\right)$. A simple calculation yields

$$
L(T(h))=L(T(g)) /\|g\| \leq\|T(g)\| /\|g\|=1 .
$$

Next we show that $\|T(h)\|_{\infty}<1+\varepsilon$. For any $y \in Y$, we have

$$
\|T(h)(y)\|=\|T(g /\|g\|)(y)+\varepsilon e\| \leq 1+\varepsilon
$$

since $\|T(g /\|g\|)(y)\| \leq\|T(g)\| /\|g\|=1$. Indeed,

$$
\|T(g /\|g\|)(y)+\varepsilon e\|<1+\varepsilon .
$$

Otherwise the vector $u=(1 /(1+\varepsilon))(T(g /\|g\|)(y)+\varepsilon e)$ would be an extreme point of $B_{E}$ by the strict convexity of $E$, and since $u$ is a convex combination of $T(g /\|g\|)(y)$ and $e$, which are in $B_{E}$, we infer that $T(g /\|g\|)(y)=e$, a contradiction. Hence $\|T(h)(y)\|<1+\varepsilon$ for all $y \in Y$. Since $\|T(h)\|_{\infty}=\|T(h)(y)\|$ for some $y \in Y$, we conclude that $\|T(h)\|_{\infty}<1+\varepsilon$. From what we have proved above it is deduced that $\|T(h)\|<1+\varepsilon$, but, on the other hand,

$$
1+\varepsilon=\|g(x) /\| g\|+\varepsilon e\|=\|h(x)\| \leq\|h\|_{\infty} \leq\|h\|=\|T(h)\|,
$$

which is impossible. This proves our claim.

Now, let $y \in Y$ be such that $T(g /\|g\|)(y)=e$. Since $e=g(x) /\|g\|, T g(y)=$ $g(x)$, that is,

$$
\|f\|_{\infty} T f(y)+\|f\|^{2} T\left(1_{X} \otimes e\right)(y)=\left(\|f\|_{\infty}^{2}+\|f\|^{2}\right) e .
$$

Since $T\left(1_{X} \otimes e\right)=1_{Y} \otimes e$, we have

$$
\|f\|_{\infty} T(f)(y)+\|f\|^{2} e=\left(\|f\|_{\infty}^{2}+\|f\|^{2}\right) e,
$$

and thus $T(f)(y)=\|f\|_{\infty} e$, which is $T(f)(y)=f(x)$ since $f \in F(x)$. Hence $P(f) \neq \emptyset$. Moreover, $P(f)$ is closed in $Y$ since $P(f)=T(f)^{-1}(\{f(x)\})$ and $T(f)$ is continuous.

Step 2. For each $x \in X$, the set

$$
B(x)=\{y \in Y: T(f)(y)=f(x), \forall f \in F(x)\}
$$

is nonempty and closed. 
Let $x \in X$. For each $f \in F(x), P(f)$ is a nonempty closed subset of $Y$ by Step 1. Since $B(x)=\bigcap_{f \in F(x)} P(f), B(x)$ is closed. To prove that $B(x) \neq \emptyset$, since $Y$ is compact and $B(x)=\bigcap_{f \in F(x)} P(f)$, it suffices to check that if $f_{1}, \ldots, f_{n} \in F(x)$, then $\bigcap_{j=1}^{n} P\left(f_{j}\right) \neq \emptyset$.

We can suppose, without loss of generality, that $f_{j} \neq 0$ for all $j \in\{1, \ldots, n\}$ since $P\left(f_{j}\right)=Y$ if $f_{j}=0$. For each $j \in\{1, \ldots, n\}$ define $g_{j}=\left\|f_{j}\right\|_{\infty} f_{j}+\left\|f_{j}\right\|^{2}\left(1_{X} \otimes e\right)$. As in the proof of Step 1, $g_{j} \in \operatorname{Lip}(X, E)$ with $g_{j}(x)=\left(\left\|f_{j}\right\|_{\infty}^{2}+\left\|f_{j}\right\|^{2}\right) e$ and $\left\|g_{j}\right\|=\left\|f_{j}\right\|_{\infty}^{2}+\left\|f_{j}\right\|^{2}$. Hence $g_{j} \neq 0$ and we can define $h=(1 / n) \sum_{j=1}^{n}\left(g_{j} /\left\|g_{j}\right\|\right)$. Clearly, $h \in \operatorname{Lip}(X, E), h(x)=e$ and $\|h\|_{\infty}=1$. Hence $h(x)=\|h\|_{\infty} e$ and thus $h \in F(x)$. Then, by Step 1, there exists a point $y \in Y$ such that $T(h)(y)=h(x)$. Since $T(h)(y)=(1 / n) \sum_{j=1}^{n}\left(T\left(g_{j}\right)(y) /\left\|g_{j}\right\|\right)$ and $h(x)=e$, it follows that $e=$ $(1 / n) \sum_{j=1}^{n}\left(T\left(g_{j}\right)(y) /\left\|g_{j}\right\|\right)$. Since $E$ is strictly convex and $\left\|T\left(g_{j}\right)(y)\right\| /\left\|g_{j}\right\| \leq$ $\left\|T\left(g_{j}\right)\right\| /\left\|g_{j}\right\|=1$ for all $j \in\{1, \ldots, n\}$, we infer that $T\left(g_{j}\right)(y)=\left\|g_{j}\right\| e$ for all $j \in$ $\{1, \ldots, n\}$. Reasoning as in Step 1, we obtain $T\left(f_{j}\right)(y)=f_{j}(x)$ for all $j \in\{1, \ldots, n\}$ and thus $y \in \bigcap_{j=1}^{n} P\left(f_{j}\right)$.

Step 3. Let $f \in \operatorname{Lip}(X, E), x \in X$ and $y \in B(x)$. If $f(x)=0$, then $T(f)(y)=0$.

If $f=0$, then there is nothing to prove. Suppose $f \neq 0$ and let $\delta=\|f\|_{\infty} /\|f\|$. Clearly, $L(f) /\|f\|_{\infty} \leq 1 / \delta$. Consider $h_{x, \delta} \otimes e \in F(x)$. We next prove that $f /\|f\|_{\infty}+\left(h_{x, \delta} \otimes e\right)$ belongs to $F(x)$. Since $f /\|f\|_{\infty}+\left(h_{x, \delta} \otimes e\right) \in \operatorname{Lip}(X, E)$ and $f(x) /\|f\|_{\infty}+\left(h_{x, \delta} \otimes e\right)(x)=e$, it suffices to check that $\|f /\| f\left\|_{\infty}+\left(h_{x, \delta} \otimes e\right)\right\|_{\infty}=$ 1. Let $z \in X$. If $d(z, x) \geq \delta$, we have $\left(h_{x, \delta} \otimes e\right)(z)=0$ and so

$$
\|f(z) /\| f\left\|_{\infty}+\left(h_{x, \delta} \otimes e\right)(z)\right\|=\|f(z)\| /\|f\|_{\infty} \leq 1 .
$$

If $d(z, x)<\delta$, then $\left(h_{x, \delta} \otimes e\right)(z)=(1-d(z, x) / \delta) e$, and therefore

$$
\|f(z) /\| f\left\|_{\infty}+\left(h_{x, \delta} \otimes e\right)(z)\right\| \leq\|f(z)\| /\|f\|_{\infty}+1-d(z, x) / \delta \leq 1
$$

since

$$
\|f(z)\| /\|f\|_{\infty}=\|f(z)-f(x)\| /\|f\|_{\infty} \leq L(f) d(z, x) /\|f\|_{\infty} \leq d(z, x) / \delta .
$$

Hence $\|f /\| f\left\|_{\infty}+\left(h_{x, \delta} \otimes e\right)(z)\right\|_{\infty} \leq 1$. Since

$$
\|f(x) /\| f\left\|_{\infty}+\left(h_{x, \delta} \otimes e\right)(x)\right\|=\|e\|=1
$$

we obtain the desired condition.

By the definition of $B(x)$ it follows that

$$
T\left(f /\|f\|_{\infty}+\left(h_{x, \delta} \otimes e\right)\right)(y)=\left(f /\|f\|_{\infty}+\left(h_{x, \delta} \otimes e\right)\right)(x)
$$

that is, $T(f)(y) /\|f\|_{\infty}+T\left(h_{x, \delta} \otimes e\right)(y)=e$. Moreover, since $y \in B(x)$ and $h_{x, \delta} \otimes e \in$ $F(x)$, we have $T\left(h_{x, \delta} \otimes e\right)(y)=\left(h_{x, \delta} \otimes e\right)(x)=e$. Hence $T(f)(y) /\|f\|_{\infty}+e=e$ and thus $T(f)(y)=0$.

Step 4. Let $x, x^{\prime} \in X$ with $x \neq x^{\prime}$. Then $B(x) \cap B\left(x^{\prime}\right)=\emptyset$.

Suppose $y \in B(x) \cap B\left(x^{\prime}\right)$. Let $\delta=d\left(x, x^{\prime}\right)>0$ and consider $h_{x, \delta} \otimes e$. Since $y \in B(x)$ and $h_{x, \delta} \otimes e \in F(x)$, we have $T\left(h_{x, \delta} \otimes e\right)(y)=\left(h_{x, \delta} \otimes e\right)(x)=e$ by Step 2 but Step 3 also yields $T\left(h_{x, \delta} \otimes e\right)(y)=0$ since $y \in B\left(x^{\prime}\right)$ and $\left(h_{x, \delta} \otimes e\right)\left(x^{\prime}\right)=0$. So we arrive at a contradiction. Hence $B(x) \cap B\left(x^{\prime}\right)=\emptyset$.

Steps 3 and 4 motivate the following:

Definition 1. Let $Y_{0}=\bigcup_{x \in X} B(x)$. Define $\varphi: Y_{0} \rightarrow X$ by $\varphi(y)=x$ if $y \in B(x)$. 
Clearly, $\varphi$ is surjective. Moreover, given $y \in Y_{0}$, there exists $x \in X$ such that $y \in B(x)$, and hence $\varphi(y)=x$ and $T(f)(y)=f(x)$ for all $f \in F(x)$.

We shall obtain the representation of $T$ in terms of the following functions.

Definition 2. For each $y \in Y$, define $T_{y}: E \rightarrow E$ by $T_{y}(u)=T\left(1_{X} \otimes u\right)(y)$.

It is easy to show that $T_{y} \in L(E)$ with $\left\|T_{y}\right\|=1=\left\|T_{y}(e)\right\|$ for all $y \in Y$.

Step 5. The map $y \mapsto T_{y}$ from $Y$ into $L(E)$ is Lipschitz.

Let $y, z \in Y$. Given $u \in E$, we have

$$
\begin{aligned}
\left\|\left(T_{y}-T_{z}\right)(u)\right\| & \leq L\left(T\left(1_{X} \otimes u\right)\right) d(y, z) \\
& \leq\left\|T\left(1_{X} \otimes u\right)\right\| d(y, z)=\|u\| d(y, z),
\end{aligned}
$$

and thus $\left\|T_{y}-T_{z}\right\| \leq d(y, z)$.

Step 6. $T(f)(y)=T_{y}(f(\varphi(y)))$ for all $f \in \operatorname{Lip}(X, E)$ and $y \in Y_{0}$.

Let $f \in \operatorname{Lip}(X, E)$ and $y \in Y_{0}$. Let $x=\varphi(y) \in X$ and define $h=f-\left(1_{X} \otimes f(x)\right)$. Obviously, $h \in \operatorname{Lip}(X, E)$ with $h(x)=0$. From Step 3, we have $T(h)(y)=0$ and therefore $T(f)(y)=T\left(1_{X} \otimes f(x)\right)(y)=T_{y}(f(x))=T_{y}(f(\varphi(y)))$.

Step 7. $Y_{0}$ is closed in $Y$.

Let $y \in Y$ and let $\left\{y_{n}\right\}$ be a sequence in $Y_{0}$ which converges to $y$. Let $x_{n}=\varphi\left(y_{n}\right)$ for all $n \in \mathbb{N}$. Since $X$ is compact, there exists a subsequence $\left\{x_{\sigma(n)}\right\}$ converging to a point $x \in X$. Let $f \in F(x)$. Clearly, $\left\{T(f)\left(y_{\sigma(n)}\right)\right\}$ converges to $T(f)(y)$, but also to $f(x)$ as we see at once. Indeed, for each $n \in \mathbb{N}$, we have

$$
T(f)\left(y_{\sigma(n)}\right)=T_{y_{\sigma(n)}}\left(f\left(x_{\sigma(n)}\right)\right)=T\left(1_{X} \otimes f\left(x_{\sigma(n)}\right)\right)\left(y_{\sigma(n)}\right),
$$

by Step 6, and

$$
\begin{aligned}
f(x) & =\|f\|_{\infty} e=\|f\|_{\infty}\left(1_{Y} \otimes e\right)\left(y_{\sigma(n)}\right) \\
& =\|f\|_{\infty} T\left(1_{X} \otimes e\right)\left(y_{\sigma(n)}\right)=T\left(1_{X} \otimes f(x)\right)\left(y_{\sigma(n)}\right),
\end{aligned}
$$

since $f \in F(x)$. We deduce that

$$
\begin{aligned}
\left\|T(f)\left(y_{\sigma(n)}\right)-f(x)\right\| & =\left\|T\left(1_{X} \otimes\left(f\left(x_{\sigma(n)}\right)-f(x)\right)\right)\left(y_{\sigma(n)}\right)\right\| \\
& \leq\left\|T\left(1_{X} \otimes\left(f\left(x_{\sigma(n)}\right)-f(x)\right)\right)\right\|=\left\|1_{X} \otimes\left(f\left(x_{\sigma(n)}\right)-f(x)\right)\right\| \\
& =\left\|f\left(x_{\sigma(n)}\right)-f(x)\right\|
\end{aligned}
$$

for all $n \in \mathbb{N}$. Since $\left\{f\left(x_{\sigma(n)}\right)\right\} \rightarrow f(x)$, we conclude that $\left\{T(f)\left(y_{\sigma(n)}\right)\right\} \rightarrow f(x)$. Hence $T(f)(y)=f(x)$ and thus $y \in B(x) \subset Y_{0}$.

Step 8. The map $\varphi: Y_{0} \rightarrow X$ is Lipschitz and $L(\varphi) \leq \max \{1, \operatorname{diam}(X) / 2\}$.

Let $y, z \in Y_{0}$ be such that $\varphi(y) \neq \varphi(z)$ and put $\delta=d(\varphi(y), \varphi(z)) / 2$. Define $f_{y, z}=\delta\left(h_{\varphi(y), \delta}-h_{\varphi(z), \delta}\right)$ on $X$. It is easy to see that $f_{y, z} \in \operatorname{Lip}(X)$ and $\left\|f_{y, z}\right\| \leq k:=\max \{1, \operatorname{diam}(X) / 2\}$. Since $T$ is an isometry, $\left\|T\left(f_{y, z} \otimes e\right)\right\| \leq k$. This inequality implies $L\left(T\left(f_{y, z} \otimes e\right)\right) \leq k$. It follows that

$$
\left\|T\left(f_{y, z} \otimes e\right)(y)-T\left(f_{y, z} \otimes e\right)(z)\right\| \leq k d(y, z) .
$$

Using Step 6 we get

$$
\begin{aligned}
& T\left(f_{y, z} \otimes e\right)(y)=T_{y}\left(\left(f_{y, z} \otimes e\right)(\varphi(y))\right)=T_{y}(\delta e)=\delta e, \\
& T\left(f_{y, z} \otimes e\right)(z)=T_{z}\left(\left(f_{y, z} \otimes e\right)(\varphi(z))\right)=T_{z}(-\delta e)=-\delta e .
\end{aligned}
$$

We conclude that $d(\varphi(y), \varphi(z)) \leq k d(y, z)$. 
The condition in Theorem 2.1. $T\left(1_{X} \otimes e\right)=1_{Y} \otimes e$ for some $e \in S_{E}$, is not too restrictive if we analyse the known results in the scalar case. In this case our condition means $T\left(1_{X}\right)=1_{Y}$; notice that the connectedness assumptions on the metric spaces in [9, Lemma 1.5] and [11, Lemma 6] yield a similar condition, namely, that $T\left(1_{X}\right)$ is a constant function.

\section{A LipsChitZ VERSiON OF JERISON'S THEOREM}

Recall that a map between metric spaces $\varphi: X \rightarrow Y$ is said to be a Lipschitz homeomorphism if $\varphi$ is bijective and $\varphi$ and $\varphi^{-1}$ are both Lipschitz.

Theorem 3.1. Let $X, Y$ be compact metric spaces and let $E$ be a strictly convex Banach space. Let $T$ be a linear isometry from $\operatorname{Lip}(X, E)$ onto $\operatorname{Lip}(Y, E)$ such that $T\left(1_{X} \otimes e\right)=1_{Y} \otimes e$ for some $e \in S_{E}$. Then there exists a Lipschitz homeomorphism $\varphi: Y \rightarrow X$ with $L(\varphi) \leq \max \{1, \operatorname{diam}(X) / 2\}$ and $L\left(\varphi^{-1}\right) \leq \max \{1, \operatorname{diam}(Y) / 2\}$, and a Lipschitz map $y \mapsto T_{y}$ from $Y$ into $L(E)$ where $T_{y}$ is an isometry from $E$ onto itself for all $y \in Y$ such that

$$
T(f)(y)=T_{y}(f(\varphi(y))), \quad \forall y \in Y, \forall f \in \operatorname{Lip}(X, E) .
$$

Proof. Let $Y_{0}$ and $\varphi$ be as in Theorem 2.1. Since $T^{-1}: \operatorname{Lip}(Y, E) \rightarrow \operatorname{Lip}(X, E)$ is a linear isometry and $T^{-1}\left(1_{Y} \otimes e\right)=1_{X} \otimes e$, applying Theorem 2.1 we have

$$
T^{-1}(g)(x)=\left(T^{-1}\right)_{x}(g(\psi(x))), \quad \forall x \in X_{0}, \forall g \in \operatorname{Lip}(Y, E),
$$

where $\psi$ is a Lipschitz map from a closed subset $X_{0}$ of $X$ onto $Y$ with $L(\psi) \leq$ $\max \{1, \operatorname{diam}(Y) / 2\}$, and $x \mapsto\left(T^{-1}\right)_{x}$ is a Lipschitz map from $X$ into $L(E)$. Namely, $X_{0}=\bigcup_{y \in Y} B(y)$ where, for each $y \in Y$,

$$
B(y)=\left\{x \in X: T^{-1}(g)(x)=g(y), \forall g \in F(y)\right\}
$$

with

$$
F(y)=\left\{g \in \operatorname{Lip}(Y, E): g(y)=\|g\|_{\infty} e\right\},
$$

and $\psi: X_{0} \rightarrow Y$ is the Lipschitz map defined by $\psi(x)=y$ if $x \in B(y)$. Moreover, using the same arguments as in Step 3, the following can be proved:

Claim 1. Let $g \in \operatorname{Lip}(Y, E), y \in Y$ and $x \in B(y)$. If $g(y)=0$, then $T^{-1}(g)(x)=0$.

After this preparation we proceed to prove the theorem. Fix $x \in X$ and let $y \in B(x)$. We first prove that $x \in B(y)$. Suppose that $x \notin B(y)$. Since $B(y) \neq \emptyset$, there exists $x^{\prime} \in B(y)$ with $x^{\prime} \neq x$. Take $f \in \operatorname{Lip}(X, E)$ for which $f(x)=0$ and $f\left(x^{\prime}\right) \neq 0$. Since $y \in B(x)$ and $f(x)=0$, we have $T(f)(y)=0$ by Step 3 . Then $T^{-1}(T(f))\left(x^{\prime}\right)=0$ since $x^{\prime} \in B(y)$ by Claim 1, and thus $f\left(x^{\prime}\right)=0$, a contradiction. Therefore $x \in B(y) \subset X_{0}$ and thus $X_{0}=X$. Next we see that $Y_{0}=Y$. Let $y \in Y$. We can take a point $x \in B(y)$. As above it is proved that $y \in B(x)$ and thus $y \in Y_{0}$.

To see that $\varphi$ is a Lipschitz homeomorphism, let $y \in Y$. Then $y \in B(x)$ for some $x \in X$, that is, $\varphi(y)=x$. Moreover, by what we have proved above, $x \in B(y)$ and so $\psi(x)=y$. As a consequence, $\psi(\varphi(y))=y$. Since $\varphi$ was surjective, $\varphi$ is bijective with $\varphi^{-1}=\psi$ and thus $\varphi$ is a Lipschitz homeomorphism.

To check that $T_{y}$ is an isometry from $E$ into itself for every $y \in Y$, we first show that $T$ sends nonvanishing functions of $\operatorname{Lip}(X, E)$ into nonvanishing functions of $\operatorname{Lip}(Y, E)$. Assume there exists $f \in \operatorname{Lip}(X, E)$ such that $f(x) \neq 0$ for all $x \in X$, but $T(f)(y)=0$ for some $y \in Y$. By the surjectivity of $\psi$, there is a point $x \in X_{0}$ such that $\psi(x)=y$, that is, $x \in B(y)$. Since $T(f)(y)=0$, by Claim 1 
we have $f(x)=T^{-1}(T(f))(x)=0$, a contradiction. Hence $T$ maps nonvanishing functions into nonvanishing functions. If, for some $y \in Y, T_{y}$ is not an isometry, then there exists a $u \in S_{E}$ such that $\left\|T_{y}(u)\right\|=\left\|T\left(1_{X} \otimes u\right)(y)\right\|<1$. Since $T$ is surjective, there is an $f \in \operatorname{Lip}(X, E)$ such that $T(f)=1_{Y} \otimes T\left(1_{X} \otimes u\right)(y)$. Thus $\|f\|_{\infty} \leq\|f\|=\|T(f)\|=\left\|T\left(1_{X} \otimes u\right)(y)\right\|<1$ and $\left(1_{X} \otimes u\right)-f$ never vanishes on $X$. As $T\left(1_{X} \otimes u\right)(y)=T(f)(y)$, we arrive at a contradiction.

Next we prove that $T_{y}: E \rightarrow E$ is surjective for every $y \in Y$. Fix $y \in Y$ and let $v \in E$. Since $T$ is surjective, there exists $f \in \operatorname{Lip}(X, E)$ such that $T(f)=1_{Y} \otimes v$. Let $u=(f \circ \varphi)(y) \in E$. Using Step [6 we have $T_{y}(u)=T_{y}(f(\varphi(y)))=T(f)(y)=v$. Hence $T_{y}$ is surjective.

Finally, as a direct consequence of Theorem 3.1, we obtain the following:

Corollary 3.2. Let $X, Y$ be compact metric spaces with diameter at most 2 and let $E$ be a strictly convex Banach space. Then every surjective linear isometry $T$ from $\operatorname{Lip}(X, E)$ into $\operatorname{Lip}(Y, E)$ satisfying that $T\left(1_{X} \otimes e\right)=1_{Y} \otimes$ e for some $e \in S_{E}$, can be expressed as $T(f)(y)=T_{y}(f(\varphi(y)))$ for all $y \in Y$ and $f \in \operatorname{Lip}(X, E)$, where $\varphi: Y \rightarrow X$ is a surjective isometry and $y \mapsto T_{y}$ is a Lipschitz map from $Y$ into $L(E)$ such that $T_{y}$ is an isometry from $E$ onto $E$ for all $y \in Y$.

In the special case that $E$ is a Hilbert space, Theorems 2.1 and 3.1 can be improved as follows. For a Hilbert space $E$, let us recall that a unitary operator is a linear map $\Phi: E \rightarrow E$ that is a surjective isometry.

Corollary 3.3. Let $X$ and $Y$ be compact metric spaces and let $E$ be a Hilbert space. Let $T$ be a linear isometry from $\operatorname{Lip}(X, E)$ into $\operatorname{Lip}(Y, E)$ such that $T\left(1_{X} \otimes e\right)$ is a constant function for some $e \in S_{E}$. Then there exists a Lipschitz map $\varphi$ from a closed subset $Y_{0}$ of $Y$ onto $X$ with $L(\varphi) \leq \max \{1, \operatorname{diam}(X) / 2\}$ and a Lipschitz map $y \mapsto T_{y}$ from $Y$ into $L(E)$ with $\left\|T_{y}\right\|=1$ for all $y \in Y$ such that

$$
T(f)(y)=T_{y}(f(\varphi(y))), \quad \forall y \in Y_{0}, \forall f \in \operatorname{Lip}(X, E) .
$$

If, in addition, $T$ is surjective, then $Y_{0}=Y, \varphi$ is a Lipschitz homeomorphism with $L\left(\varphi^{-1}\right) \leq \max \{1, \operatorname{diam}(Y) / 2\}$ and, for each $y \in Y, T_{y}$ is a unitary operator.

Proof. Assume that $T\left(1_{X} \otimes e\right)=1_{Y} \otimes u$ for some $u \in E$. Obviously, $\|u\|=1$. Since $E$ is a Hilbert space, we can construct a unitary operator $\Phi: E \rightarrow E$ such that $\Phi(u)=e$. Define $S: \operatorname{Lip}(Y, E) \rightarrow \operatorname{Lip}(Y, E)$ by

$$
S(g)(y)=\Phi(g(y)), \quad \forall y \in Y, \forall g \in \operatorname{Lip}(Y, E) .
$$

It is easy to prove that $S$ is a surjective linear isometry satisfying that $S\left(1_{Y} \otimes u\right)=$ $1_{Y} \otimes e$. Hence $R=S \circ T$ is a linear isometry from $\operatorname{Lip}(X, E)$ into $\operatorname{Lip}(Y, E)$ with $R\left(1_{X} \otimes e\right)=1_{Y} \otimes e$. Then Theorem 2.1 guarantees the existence of a Lipschitz map $\varphi$ from a closed subset $Y_{0}$ of $Y$ onto $X$ with $L(\varphi) \leq \max \{1, \operatorname{diam}(X) / 2\}$ and a Lipschitz map $y \mapsto R_{y}$ from $Y$ into $L(E)$ with $\left\|R_{y}\right\|=1$ for all $y \in Y$ such that

$$
R(f)(y)=R_{y}(f(\varphi(y))), \quad \forall y \in Y_{0}, \forall f \in \operatorname{Lip}(X, E) .
$$

For each $y \in Y$, consider $T_{y}=\Phi^{-1} \circ R_{y} \in L(E)$. It is easily seen that the map $y \mapsto T_{y}$ from $Y$ into $L(E)$ is Lipschitz with $\left\|T_{y}\right\|=1$ for all $y \in Y$. Moreover, for any $y \in Y_{0}$ and $f \in \operatorname{Lip}(X, E)$, we have

$$
T(f)(y)=\Phi^{-1}\left(R_{y}(f(\varphi(y)))\right)=T_{y}(f(\varphi(y))) .
$$

If, in addition, $T$ is surjective, the rest of the corollary follows by applying Theorem 3.1 to $R$. 


\section{ACKNOWLEDGEMENT}

We thank the referee for several helpful suggestions which have improved this paper, especially Corollary 3.3 .

\section{REFERENCES}

1. M. Cambern, A Holsztyński theorem for spaces of continuous vector-valued functions, Studia Math. 63 (1978), 213-217. MR515491 (80d:46049)

2. K. Jarosz and V. D. Pathak, Isometries between function spaces, Trans. Amer. Math. Soc. 305 (1988), 193-206. MR920154 (89e:46026)

3. T. M. Jenkins, Banach Spaces of Lipschitz Functions on an Abstract Metric Space, Ph.D. Thesis, Yale University, 1968.

4. M. Jerison, The space of bounded maps into a Banach space, Ann. of Math. (2) 52 (1950), 309-327. MR0036942 (12:188c)

5. A. Jiménez-Vargas and Moisés Villegas-Vallecillos, Into linear isometries between spaces of Lipschitz functions, Houston J. Math. 34 (2008), 1165-1184.

6. K. de Leeuw, Banach spaces of Lipschitz functions, Studia Math. 21 (1961/62), 55-66. MR.0140927 (25:4341)

7. E. Mayer-Wolf, Isometries between Banach spaces of Lipschitz functions, Israel J. Math. 38 (1981), 58-74. MR599476 (82e:46036)

8. W. Novinger, Linear isometries of subspaces of continuous functions, Studia Math. 53 (1975), 273-276. MR0417770 (54:5818)

9. A. K. Roy, Extreme points and linear isometries of the Banach space of Lipschitz functions, Canad. J. Math. 20 (1968), 1150-1164. MR0236685 (38:4980)

10. M. H. Vasavada, Closed Ideals and Linear Isometries of Certain Function Spaces, Ph.D. Thesis, Wisconsin University, 1969.

11. N. Weaver, Isometries of noncompact Lipschitz spaces, Canad. Math. Bull. 38 (1995), 242249. MR 1335105 (96b:46032)

12. N. Weaver, Lipschitz Algebras, World Scientific Publishing Co., River Edge, NJ, 1999. MR 1832645 (2002g:46002)

Departamento de Álgebra y AnÁlisis Matemático, Universidad de Almería, 04071, Almería, Spain

E-mail address: ajimenez@ual.es

Departamento de Álgebra y AnÁlisis Matemático, Universidad de Almería, 04071, Almería, Spain

E-mail address: mvv042@alboran.ual.es 